University of Wollongong

Research Online

Faculty of Engineering and Information

Faculty of Engineering and Information

Sciences - Papers: Part A

Sciences

$1-1-2005$

Dynamic channel allocation for mobile cellular systems using a control theoretical approach

Yaya Wei

Tsinghua University

Chuang Lin

Tsinghua University

Raad Raad

University of Wollongong, raad@uow.edu.au

Fengyuan Ren

University of Wollongong

Follow this and additional works at: https://ro.uow.edu.au/eispapers

Part of the Engineering Commons, and the Science and Technology Studies Commons

Research Online is the open access institutional repository for the University of Wollongong. For further information contact the UOW Library: research-pubs@uow.edu.au 


\title{
Dynamic channel allocation for mobile cellular systems using a control theoretical approach
}

\author{
Abstract \\ The guard channel scheme in wireless mobile networks has attracted and is still drawing research \\ interest owing to easy implementation and flexible control. Dynamic guard channel schemes have already \\ been proposed in the literature to adapt to varying traffic load. This paper presents a novel control- \\ theoretic approach to dynamically reserve guard channels called PI-Guard Channel (PI-GC) controller that \\ maintains the handoff blocking probability (HBP) to a predefined value; while it still improves the channel \\ resource utilization.

\section{Keywords} \\ dynamic, channel, allocation, theoretical, mobile, approach, cellular, systems, control

\section{Disciplines \\ Engineering | Science and Technology Studies} \\ Publication Details \\ Y. Wei, C. Lin, R. Raad \& F. Ren, "Dynamic channel allocation for mobile cellular systems using a control \\ theoretical approach," IEICE Transactions on Communications, vol. 5, (2) pp. 2741-2745, 2005.
}




\section{Dynamic Channel Allocation for Mobile Cellular Systems Using a Control Theoretical Approach}

\author{
Yaya Wei , Chuang Lin \\ Department of Computer Science, Tsinghua University \\ Beijing, China \\ \{yywei, clin\}@csnet1.cs.tsinghua.edu.cn
}

\author{
Raad Raad, Fengyuan Ren \\ University of Wollongong \\ Wollongong, Australia \\ raad@uow.edu.au; renfy@csnet1.cs.tsinghua.edu.cn
}

\begin{abstract}
The guard channel scheme in wireless mobile networks has attracted and is still drawing research interest owing to easy implementation and flexible control. However guard channel schemes can not adapt to changing traffic loads because of static reserved guard channels. Therefore dynamic guard channel schemes have been proposed in the literature to adapt to varying traffic load. This paper presents a novel controltheoretic approach to dynamically reserve guard channels called PI-Guard Channel (PI-GC) controller. Experiments show that our proposed scheme can maintain the handoff blocking probability (HBP) to a predefined value while it still improves the channel resource utilization.
\end{abstract}

Keywords- Handoff, guard channel, resource utilization, PI controller

\section{INTRODUCTION}

$\mathrm{H}$ andoff procedures are very important in maintaining

service continuity and QoS guarantees to mobile applications. The Handoff Blocking Probability (HBP) is the main performance metric measuring the continuity of a call while the new call blocking probability (NBP) is another important parameter measuring connectivity. These two parameters combined with resource utilization form the Grade of Service (GOS) measure of a mobile network.

Many different admission control strategies have been discussed in literature to improve these three important parameters. These strategies can be classified into two categories: Guard Channel (GC) schemes [1] and the shadow cluster concept [2]. In the GC scheme, a set of channels are permanently reserved exclusively for handoff calls to keep HBP lower than a predefined value. However, the static reservation in the GC schemes is not efficient for varying traffic conditions found in wireless networks. Recently, several dynamic reservation schemes proposed calculating the required bandwidth in order to maintain a low HBP [3][9][10]. However, the proposed heuristic algorithm in [3], which is independent of any model, cannot guarantee the HBP to the predefined values. [9][10] proposed the statistical models to

This work is supported by the National Natural Science Foundation of China (No.60429202, 90412012 and 60373013), NSFC and RGC (No. 60218003), the National Grand Fundamental Research 973 Program of China (No.2003CB314804) estimate the resource requirements for handoff calls, but statistical model was not realistic. The other reservation scheme is the concept of the shadow cluster, which proposes to reduce the call blocking probability by predictive resource allocation. In this scheme, a shadow cluster represents a set of cells around an active mobile. A decision is made on how much bandwidth will be reserved in the shadow cluster of cells depending on the mobiles' velocity. The amount of reserved bandwidth in each cell is reduced as the mobile move further and further away from the cell. However, determination of the shadow cluster always requires each base station in the shadow cluster to predict future resource demands according to the information about active mobile users' bandwidth requirements, position, movement pattern, and time. Consequently, it is computationally too expensive to be practical. Also, the decisions made are formed with the assumption that collected information is accurate and timely. Given the number of variables, there is a danger that the information may become obsolete quickly leading to inaccurate reservations in the shadow cluster. Comparably, the GC scheme is the simpler and more flexible scheme to give priority to handoff calls. The key issue is how to dynamically reserve guard channels to adapt to varying loads.

Unlike the heuristic algorithms that are independent of the system model in previous works, a PI controller based on the proposed approximate model for HBP has been proposed to guarantee HBP in this paper. As the best of author's knowledge, this is the first time to use a control-theoretic controller for handoff scheme. Actually, the control-theoretic controller has been an active area of research with various applications. For example, Abdelzaher et al. [7] designed a PI controller to control the relative delay of web servers. The controller can guarantee the delay to the desired value when the traffic load varies. Chang et al. [8] used a robust nonlinear PI controller for improving AQM performance. The proposed controller can achieve the tradeoff between responsiveness and high link utilization over a large range of uncertainties by varying the control parameters according to the system state.

The main contributions of the proposed controller in our paper are highlighted as follows.

- We develop the approximate non-linear handoff blocking probability model, and convert to a linear system via small signal theory.

- We apply the well developed tuning rules to design the 


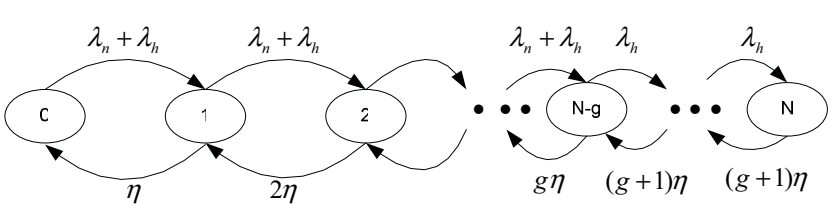

Figure 1. Markov chain model of wireless handoff

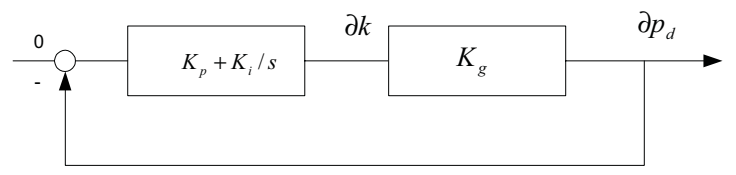

Figure 2. Block-diagram of the linearized system

PI controller for the linear system.

- The proposed controller ensures the HBP to the target value and does not reserve superfluous channels so that minimize NBP (improves the resource utilization). It adapts to varying traffic load and does not require advance knowledge or prediction regarding calls' rates.

The remainder of the paper is organized as follows: In section 2, the system model is introduced. Section 3 describes the proposed PI controller design. In section 4 , the validity of our proposed PI scheme is verified and the performance is compared with classical guard channel scheme through simulation experiments. Finally, conclusions are drawn in section 5 .

\section{MODELLING}

\section{A. HBP model}

Consider a single cell with a fixed amount of bandwidth capacity of $\mathrm{N}$ channels. Assume that the arrival stream of new calls is poissonian with the mean rate $\lambda_{n}$ and the poissonnian stream of handoff arrivals at the rate $\lambda_{h}$. The cell residence time for each call is exponentially distributed with mean $1 / \eta$. Each call requires one unit channel bandwidth. The number of guard channels is $g>0$. Let $N_{1}=N-g$ be the number of shared channels. Based on the above assumptions, a cell deploying the guard channel scheme can be modeled by a $\mathrm{M} / \mathrm{M} / \mathrm{C} / \mathrm{C}$ queuing system with the threshold state $N$-g, as illustrated in Fig. 1.

Then we can have the expression for HBP, which is the probability that all $\mathrm{N}$ channels are occupied, i.e.,

$$
P_{d}=\frac{\frac{\left(\frac{\lambda_{n}+\lambda_{h}}{\eta}\right)^{N-g}\left(\frac{\lambda_{n}}{\eta}\right)^{g}}{N !}}{\sum_{n=0}^{N-g-1} \frac{\left(\frac{\lambda_{n}+\lambda_{h}}{\eta}\right)^{n}}{n !}+\sum_{n=N-g}^{N} \frac{\left(\frac{\lambda_{n}+\lambda_{h}}{\eta}\right)^{n-g}\left(\frac{\lambda_{n}}{\eta}\right)^{n-(N-g)}}{n !}}
$$

and the recursive formula $P_{d}\left(N_{1}+k, k\right)$ is [4]:

$$
P_{d}\left(N_{1}+k, k\right)=\frac{P_{d}\left(N_{1}+(k-1), k-1\right)}{P_{d}\left(N_{1}+(k-1), k-1\right)+\frac{N}{\alpha A}} \quad k=1,2, \ldots, g
$$

where $P_{d}$ denotes the HBP, $A_{1}=a A=\lambda_{h} / \eta$ is the traffic in Erlangs due to handoff arrivals. Then we have:

$$
\frac{1}{P_{d}\left(N_{1}+k, k\right)}-\frac{1}{1-\frac{N}{\alpha A}}=\frac{N}{\alpha A}\left(\frac{1}{P_{d}\left(N_{1}+k-1, k-1\right)}-\frac{1}{1-\frac{N}{\alpha A}}\right)
$$

Since $P_{d}\left(N_{1}+k, k\right)$ is a geometric sequence, we can write the expression for $P_{d}\left(N_{1}+k, k\right)$ as:

$$
\begin{aligned}
& P_{d}\left(N_{1}+k, k\right)=\frac{\frac{1}{1-\frac{N}{\alpha A}}+\left(\frac{N}{\alpha A}\right)^{k}\left[\frac{1}{P_{d}\left(N_{1}, 0\right)}-\frac{1}{1}\right.}{\text { as } \quad P_{d}\left(N_{1}, 0\right)=P_{d}(N-k, 0)=E_{B}(A, N-k)} \\
& E_{B}(A, N-k)=\frac{\frac{A^{N}}{N !}}{1+A+\frac{A^{2}}{2 !}+\ldots \frac{A^{N-k}}{(N-k) !}}
\end{aligned}
$$

Substituting (2) and (3) into (1) yields

$$
=\frac{P_{d}\left(N_{1}+k, k\right)}{\frac{1}{1-\frac{N}{a A}}+\left(\frac{N}{a A}\right)^{k}\left[\frac{1+A+\frac{A^{2}}{2 !}+\ldots+\frac{A^{N-k}}{(N-k) !}}{\frac{A^{N-k}}{(N-k) !}}-\frac{1}{1-\frac{N}{a A}}\right]}
$$

Using the Stirling equation which can give an approximate value for the function $\mathrm{n}$ !,

$$
n ! \approx \sqrt{2 \pi} n^{n+1 / 2} e^{-n}
$$

because

$$
e^{x}=1+x+\frac{x^{2}}{2 !}+\ldots
$$

we can get the approximated expression

$$
1+A+\frac{A^{2}}{2 !}+\ldots+\frac{A^{N-k}}{(N-k) !} \approx e^{A}
$$

Using Equations (4)-(6), we can write the approximated model for $P_{d}\left(N_{l}+k, k\right)$

$$
\begin{aligned}
& P_{d}\left(N_{1}+k, k\right) \\
& \approx \frac{1}{1-\frac{N}{\alpha A}}+\left(\frac{N}{\alpha A}\right)^{k}\left[\frac{e^{A} \sqrt{2 \pi}(N-k)^{(N-k)+1 / 2} e^{-(N-k)}}{A^{N-k}}-\frac{1}{\left.1-\frac{N}{\alpha A}\right]}\right.
\end{aligned}
$$




\section{B. Linearization}

We can linearize equation (7) via small signal theory to get the linearized system. The linearization process is taken as follows. Taking $k$ as the input, it is assumed that there is an operating point $k_{0}$ in the system, which achieves $P_{d}\left(N_{1}+k_{0}, k_{0}\right)=P_{r e f}$, where $P_{\text {ref }}$ is the desired HBP. Equation (7) can be linearized about the operating point using small signal theory as follows. The linearization is a simple gain function, which is the derivative of the expression for the HBP with respect to $k$, evaluated at $k_{0}$, i.e.,

$$
\begin{aligned}
& \left.\frac{\partial P_{d}\left(N_{1}+k, k\right)}{\partial k}\right|_{k=k_{0}}= \\
& -\left[\frac{1}{\frac{1}{1-\frac{N}{a A}}+\left(\frac{N}{\alpha A}\right)^{k}\left[\frac{e^{A} \sqrt{2 \pi}(N-k)^{(N-k)+1 / 2} e^{-(N-k)}}{A^{N-k}}-\frac{1}{1-\frac{N}{\alpha A}}\right]}\right]^{2} \\
& \left\{\ln \left(\frac{N}{\alpha A}\right)\left(\frac{N}{\alpha A}\right)^{k}\left[\frac{e^{A} \sqrt{2 \pi}(N-k)^{(N-k)+1 / 2} e^{-(N-k)}}{A^{N-k}}-\frac{1}{1-\frac{N}{\alpha A}}\right]\right. \\
& +\left(\frac{N}{\alpha A}\right)^{k}\left[\frac{e^{A} \sqrt{2 \pi}(N-k)^{(N-k)+1 / 2} e^{-(N-k)}}{A^{N-k}}(\ln A-\ln (N-k)\right. \\
& \left.\left.\left.-\frac{1}{2(N-k)}\right)\right]\right\}\left.\right|_{k=k_{0}} \\
& =-P_{r e f}^{2}\left\{\ln \left(\frac{N}{a A}\right)\left(\frac{1}{P_{r e f}}-\frac{1}{1-\frac{N}{\alpha A}}\right)+\left(\ln A-\ln \left(N-k_{0}\right)-\right.\right. \\
& \left.\left.\frac{1}{2\left(N-k_{0}\right)}\right)\left(\frac{1}{P_{r e f}}-\frac{1}{1-\frac{N}{\alpha A}}+\frac{\left(\frac{N}{\alpha A}\right)^{k_{0}}}{1-\frac{N}{\alpha A}}\right)\right\}
\end{aligned}
$$

where

$$
\begin{aligned}
& \delta P_{d}\left(N_{1}+k\right)=P_{d}\left(N_{1}+k, k\right)-P_{r e f} \\
& \delta k=k-k_{0}
\end{aligned}
$$

The linearized closed loop system model is then depicted in Fig. 2. Where $K_{g}$ denotes a simplifying element and is given by

$$
K_{g}=\left.\frac{\delta P_{d}\left(N_{1}+k, k\right)}{\delta k}\right|_{k=k_{0}}
$$

\section{DESIGN OF THE CONTROLLER}

We control $\partial k$, the deviation of $k$ from the equilibrium value $k_{0}$, and our feedback signal is $\delta P_{d}$, again the deviation of $P_{d}$ for the equilibrium value $P_{\text {ref. }}$. The feedback signal can be provided by a controller, we choose the PI controller [5] for our system.

The PI controller has a transfer function given by

$$
P I(s)=K_{p}+\frac{K_{i}}{s}
$$

in the frequency domain. The PI controller can control the HBP to any specific value, which achieves zero steady state error. The design process is as follows. Our controller should satisfy the following two constraints.

1) First, the time constant of the controller is assumed to be 10 seconds. This results in fixing the closed loop pole at 0.1 .

2) Next we provide a phase margin of 45 degree. Phase margin reflects the relative stability of the controller: the amount of deviation the controller can tolerate in the system parameters before instability sets in.

The above two constraints together fix the parameters of the base controller. We keep a large phase margin because we do not want our controller to be too aggressive. The open loop transfer function is:

$$
L(s)=K_{g}\left(K_{p}+\frac{K_{i}}{s}\right)
$$

Choosing a phase-margin of 45 degree we have:

$$
K_{p}=\left|\frac{K_{i}}{\omega}\right|
$$

At this point, the following equation should also be satisfied:

$$
|L(j \omega)|=1
$$

Consequently,

$$
K_{g} \sqrt{K_{p}{ }^{2}+\frac{K_{i}^{2}}{\omega^{2}}}=1
$$

Using Equations (12)-(13), the following can be obtained:

$$
\begin{gathered}
K_{p}=\frac{1}{\sqrt{2} K_{g}} \\
K_{i}=-V_{\text {pole }}\left(K_{p}+\frac{1}{K_{g}}\right)
\end{gathered}
$$

where $V_{\text {pole }}$ is the value of the closed loop pole we have chosen.

The above design process yields a design in the continuous domain; however it must be implemented digitally. Therefore Equation (10) is converted to discrete form by using a bilinear transform. The bilinear transform [11] is operated by:

$$
s=>\frac{2 f_{T}(z-1)}{z+1}
$$

where $f_{T}$ is the sampling frequency. Then we get the following parameters in the discrete domain:

TABLE I.

$K_{0}$ UNDER DIFFERENT TRAFFIC LOADS

\begin{tabular}{|l|c|c|c|c|c|c|c|}
\hline $\boldsymbol{A}_{\boldsymbol{I}}$ & $\mathbf{2 0}$ & $\mathbf{3 0}$ & $\mathbf{4 0}$ & $\mathbf{5 0}$ & $\mathbf{6 0}$ & $\mathbf{7 0}$ & $\mathbf{8 0}$ \\
\hline$k_{0}$ & 2 & 3 & 4 & 5 & 7 & 9 & 13 \\
\hline
\end{tabular}




$$
\begin{gathered}
g^{\prime}=K_{p}+\frac{K_{i}}{2 f_{T}} \\
r=K_{p}-\frac{K_{i}}{2 f_{T}}
\end{gathered}
$$

Therefore the discrete form of the PI control function is:

$$
k(m)=-\left(g^{\prime}\left(P_{d}(m)-P_{r e f}\right)-r\left(P_{d}(m-1)-P_{r e f}\right)\right)+k(m-1)
$$

If $A_{1}$ and $A$ are given, $k_{0}$ is computed by Equation (4) which satisfies $P_{d}\left(N_{1}+k_{0}, k_{0}\right)=P_{\text {ref. }}$. Assume the capacity of a single cell $(N)$ is 100 , the possible traffic load of handoff calls in realistic environment $\left(A_{1}\right)$ is $10-80$ Erlangs. The traffic load of new calls is assumed to be 100 Erlangs. If $A_{1}, A$ and $k_{0}$ are given, the value of $K_{g}$ can be computed by Equation (9). Table 1 and Figure 3 show the value of $k_{0}$ and $K_{g}$ respectively.

It can be seen from Table 1 that $k_{0}$ increases to meet the target HBP when handoff call traffic is increasing. However, $K_{g}$ changes little when the handoff call traffic varies. On the other hand, the system is absolutely stable whenever $K_{g}$ varies under different traffic loads. The reason is that the only pole of the characteristic equation of the system, which is $-\frac{K_{g} K_{i}}{1+K_{g} K_{p}}$, is negative in all scenarios. For the convenience of analysis and design, we set $K_{g}$ as the value in the typical scenario, thus $N=100, A=90, A_{1}=30, K_{g}=-0.011$. According to this nominal value, the controller can be designed. Note that the controller using this approach can work steadily under various traffic loads, which is guaranteed by the stability margin of the controller.

\section{SimUlations}

In this section, the performance of the proposed PI-GC scheme is evaluated via simulations and compared with the GC scheme. The simulations are conducted using SMPL [6], which is a discrete event simulator. We implemented the PIGC and GC schemes and compared their performance under different scenarios. Our goal is to investigate whether the HBP target value can be met and the effect on cell utilization.

\section{A. System under High Load}

To simulate a system under high load, system parameters were set as follows. The capacity of each cell is $N=100$ channels. The mean call arrival rates of new calls and handoff calls are $\lambda_{n}=200 /$ cell/unit time and $\lambda_{h}=140 /$ cell/unit time respectively. The mean of the exponentially distributed cellresidence time $1 / \eta=0.5$ units of time. Therefore, the new call traffic is 100 Erlangs and the handoff call traffic is 70 Erlangs. The target HBP $P_{r e f}=0.01$. The sampling unit is 20 units of time. Initially the guarded channels are $k=5$. The controller was turned on at the $2^{\text {nd }}$ sampling unit to avoid the warm up phase. The results are shown in Fig. 4. The X-axis represents the sampling units.

It can be seen in Fig. 4 that the initial state HBP was 0.062 , far above the reference $P_{r e f}=0.01$ which was caused by the improper reserved number of guard channels. Then the PI controller reacted to the high load by reserving more channels for handoff calls after the controller was turned on. From Fig. 4

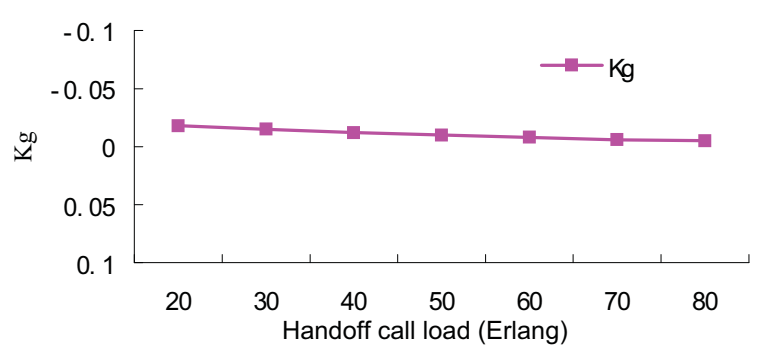

Figure $3 . K_{g}$ under different traffic loads.

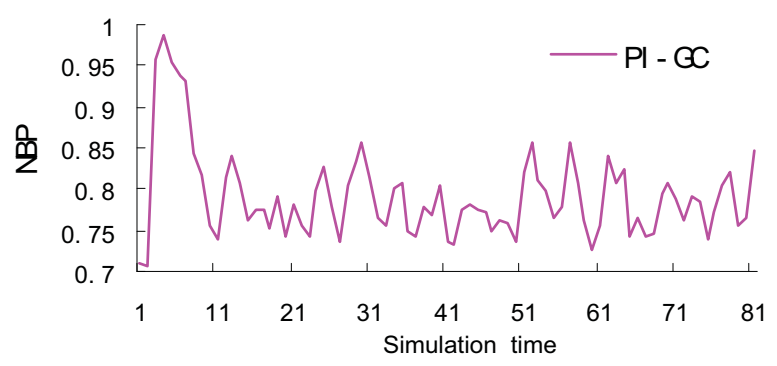

(a)

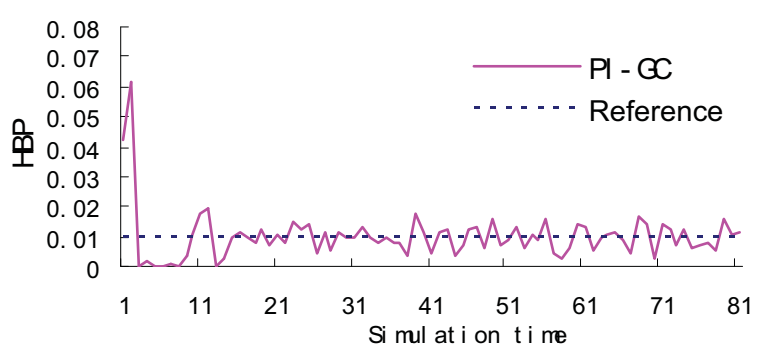

(b)

Figure 4. Performance under high load. (a) NBP and (b) HBP.

(b), it can be seen that $P_{d}$ dropped to 0.01 at the 4th sampling period. Note that the peak is reasonable because the HBP is far above the reference in the initial state. At the same time, it can be seen from Fig. 4(a) that the NBP was increased from 0.70 to 0.81 due to high new class traffic and more channels were allocated to handoff calls. After HBP converged, the system can keep HBP close to the reference.

\section{B. Varying Traffic Loads}

The QoS metrics comparison between PI-GC and GC scheme (10\% reserved channels) in terms of HBP, NBP and channel utilization is shown in Fig. 5 respectively. The $\mathrm{X}$-axis represents the handoff call traffic in Erlangs. The Y-axis represents QoS metrics when handoff traffic varied from 20 Erlangs (medium load) to 80 Erlangs (extremely high load). The new call traffic load is 100 Erlangs.

It can be seen in Fig. 5 that with PI-GC scheme HBP remained very close (within 5\%) to the reference point. The proposed PI-GC scheme, which aims at achieving the desired HBP and minimizing NBP, can reserve enough channels but not reserve superfluous channels to handoff calls. Comparably, the GC scheme reserved too many channels 


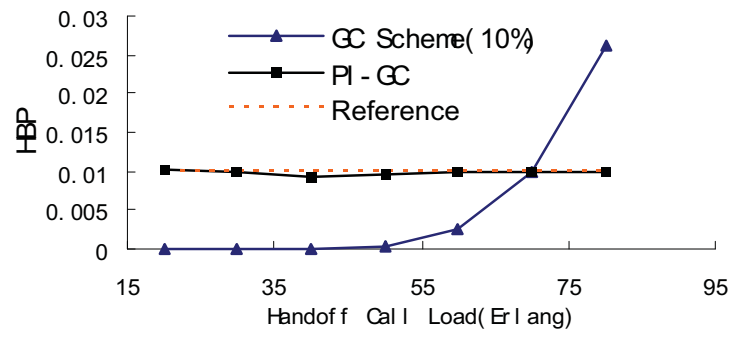

(a)

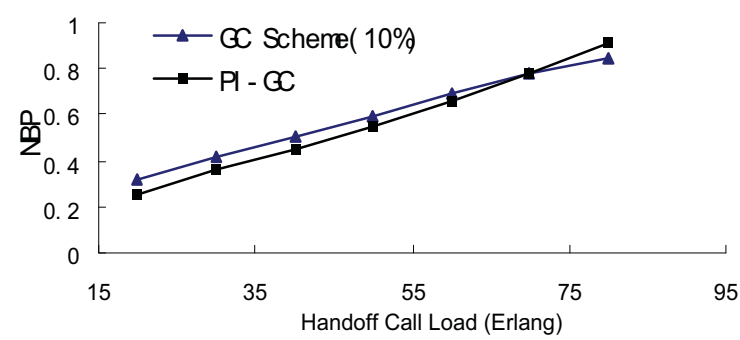

(b)

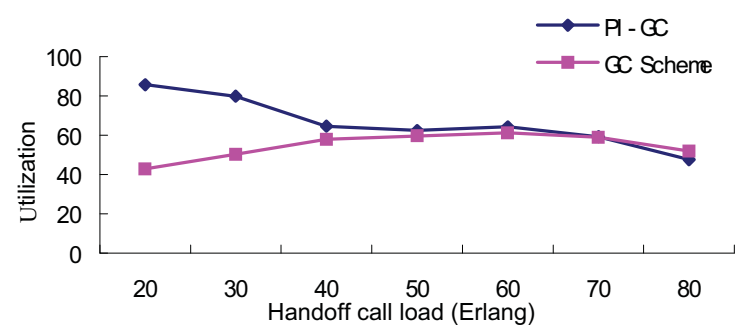

(c)

Figure 5. Performance under different traffic loads. (a) HBP (b)NBP (c) Utilization

when the traffic load is medium (20 45 Erlangs). This is also the reason why the system utilization in GC is much lower than the utilization in the proposed PI-GC scheme. But when traffic load is high (75 Erlangs), the reserved channels are not sufficient to guarantee the HBP. In conclusion, our proposed PI-GC scheme can guarantee the HBP to the target value while still minimizing NBP (maximizing channel utilization).

\section{CONCLUSION}

In this paper we present the first attempt to use a PI controller to reserve guard channels, which can guarantee the HBP to the target value with varying traffic load. The primary contribution of this paper is obtaining the approximate nonlinear model and converting it to a linear system via smallsignal linearization and subsequently designing the PI controller. Experimental results demonstrate that our proposed controller is able to ensure the HBP target is met while still maximizing the resource utilization in both high loads and varying traffic loads environments.

In future work, we will try to use other controllers to dynamically reserve guard channels, such as a fuzzy controller, and compare its performance with the PI controller proposed in this paper.

\section{REFERENCES}

[1] D. Hong and S.S. Rapport, Traffic modeling and performance analysis for cellular mobile radio telephone systems with prioritized and nonprioritized handoff procedures, IEEE Trans. Veh. Techno., vol. 35, pp77-92, 1986

[2] D. Levine, I. Akyildiz, and M. Naghshineh, A resource estimation and call admission algorithm for wireless multimedia networks using the shadow cluster concept, IEEE/ACM Trans. Networking, vol. 5, pp. 1-12, Feb. 1997.

[3] Lei Huang, Sunil Kumar, C.-C.Jay Kuo. Adaptive resource allocation for multimedia QoS management in wireless networks, IEEE Trans. Veh. Thechno., VOL. 53, 2004.

[4] G. Haring, R. Marie and K.S. Trivedi, Loss formulas and their application to optimization for cellular networks, IEEE Transactions on Vehicular Technology 50 (2001) 664-673.

[5] G. F. Franklin, J. D. Powell, and A. Emami-Naeini, Feedback Control of Dynamic System. Addison-Wesley, 1995.

[6] Teemu Kerola. SMPL Simulation Subsystem-User Guide. http://www.cs.helsinki.fi/u/kerola/sim/smpl/guide.text.

[7] T. Abdelzaher, Y. Lu, R. Zhang, D. Henriksson, Practical Application of Control Theory to Web Services, Invited paper, American Control Conference, Boston, MA, June 2004.

[8] XiaoLin Changa, Jogesh K. Muppalaa, Jen-te Yub, A Robust Nonlinear PI Controller for Improving AQM Performance. International Chamber of Commerce (ICC). 2004

[9] M. Naghshineh and S.Schwartz, Distributed call admission control in mobile/wireless networks, IEEE J. Select. Areas Commun., vol. 14, pp. 711-717, May 1996

[10] S. Wu, K. Y. M. Wong, and B. Li, A new distributed and dynamic call admission policy for mobile wireless networks with QoS guarantee, in Proc. 9th IEEE Int. Symp. Personal, Indoor and Mobile Radio Communications, vol. 1, Sept. 1998,pp. 260-264.

[11] Lawrence R. Rabiner and Bernard Gold. Theory and application of digital signal processing, Prentice-Hall, 1975. 